\title{
OBJECTIVE IDENTIFICATION OF INFORMATIVE WAVELENGTH REGIONS IN GALAXY SPECTRA
}

\author{
Ching-Wa Yip ${ }^{1}$, Michael W. Mahoney ${ }^{2}$, Alexander S. Szalay ${ }^{1,3}$, István Csabai ${ }^{4}$, \\ Tamás Budavári ${ }^{1}$, Rosemary F. G. Wyse ${ }^{1}$, and LasZlo Dobos ${ }^{4}$ \\ ${ }^{1}$ Department of Physics and Astronomy, The Johns Hopkins University, 3701 San Martin Drive, Baltimore, MD 21218, USA; \\ cwyip@pha.jhu.edu, szalay@jhu.edu \\ ${ }^{2}$ Department of Mathematics, Stanford University, Stanford, CA 94305, USA; mmahoney@cs.stanford.edu \\ ${ }^{3}$ Department of Computer Science, The Johns Hopkins University, 3400 N. Charles Street, Baltimore, MD 21218, USA \\ ${ }^{4}$ Department of Physics of Complex Systems, Eötvös Loránd University, H-1117 Budapest, Hungary \\ Received 2012 December 27; accepted 2014 February 1; published 2014 April 10
}

\begin{abstract}
Understanding the diversity in spectra is the key to determining the physical parameters of galaxies. The optical spectra of galaxies are highly convoluted with continuum and lines that are potentially sensitive to different physical parameters. Defining the wavelength regions of interest is therefore an important question. In this work, we identify informative wavelength regions in a single-burst stellar population model using the CUR Matrix Decomposition. Simulating the Lick/IDS spectrograph configuration, we recover the widely used $\mathrm{D}_{n}(4000), \mathrm{H} \beta$, and $\mathrm{H} \delta_{A}$ to be most informative. Simulating the Sloan Digital Sky Survey spectrograph configuration with a wavelength range 3450-8350 $\AA$ and a model-limited spectral resolution of $3 \AA$, the most informative regions are: first region-the $4000 \AA$ break and the $\mathrm{H} \delta$ line; second region — the Fe-like indices; third region - the $\mathrm{H} \beta$ line; and fourth region-the $\mathrm{G}$ band and the $\mathrm{H} \gamma$ line. A principal component analysis on the first region shows that the first eigenspectrum tells primarily the stellar age, the second eigenspectrum is related to the age-metallicity degeneracy, and the third eigenspectrum shows an anti-correlation between the strengths of the Balmer and the $\mathrm{Ca} \mathrm{K}$ and $\mathrm{H}$ absorptions. The regions can be used to determine the stellar age and metallicity in early-type galaxies that have solar abundance ratios, no dust, and a single-burst star formation history. The region identification method can be applied to any set of spectra of the user's interest, so that we eliminate the need for a common, fixed-resolution index system. We discuss future directions in extending the current analysis to late-type galaxies. ASCII formatted tables of the regional eigenspectra are available.
\end{abstract}

Key words: galaxies: fundamental parameters - methods: data analysis

Online-only material: color figures

\section{INTRODUCTION}

It has long been known that the diversity in spectra of galaxies is driven by the variance in their underlying physical properties, such as stellar age and metallicity. The line indices, or the relatively narrow regions of a spectrum, are isolated for the parameter sensitivities. Faber (1973) found that metallicitysensitive indices from a 10 filter photometric system correlate with the luminosity of ellipticals, giving incredible insights to the role of the gravitational potential well in the chemical evolution in those galaxies. The concept of "informative" wavelength regions had since been extended to spectroscopy. Perhaps the most well-known example is the $4000 \AA$ break (Bruzual 1983) and its correlation with the stellar age and metallicity in early-type galaxies. Worthey et al. (1994) later assembled 21 absorption indices and measured them for several hundred stars in the Galaxy, and the same set was measured by Trager et al. (1998) for several hundred globular clusters and galaxies. They are called the Lick/IDS indices (Burstein et al. 1984; Faber et al. 1985). These indices are continuum subtracted, where the continua are defined on the left and right flanks in the vicinity of the actual index wavelength range. Within an index wavelength range, one or more absorption lines, or a feature, are present. Therefore, the index strength can be a sensitive measure for specific galaxy parameters. The $\mathrm{D}_{n}$ (4000) (Balogh et al. 1999) $)^{5}$ and $\mathrm{H} \delta_{A}$ (Worthey \& Ottaviani

\footnotetext{
5 Renamed by Kauffmann et al. (2003) to be " $\mathrm{D}_{n}$ (4000)," with the subscript $n$ presumably signifying the left and right regions of influence being narrower than the previous definition by Bruzual (1983). The name that appeared in Balogh et al. (1999) is "D(4000)."
}

1997) indices were subsequently defined, and were used to constrain stellar age and the starburst mass fraction in galaxies (Kauffmann et al. 2003).

The concept of informative wavelength regions has also been used in conjunction with a popular data compression technique, the principal component analysis (PCA), to derive physical parameters of galaxies. Each spectrum in a galaxy sample is represented by the weighted sum of the same orthogonal basis, hereafter the eigenspectra (Connolly et al. 1995), and the weights are called the eigencoefficients. The lowest orders of the eigenspectra encapsulate most of the sample variance in nearby galaxies (Yip et al. 2004a), forming a subspace that lies in a higher-dimensional wavelength space. Using PCA, Wild et al. (2007) have measured, for a galaxy sample from the Sloan Digital Sky Survey (SDSS; York et al. 2000), the eigencoefficients within the rest-frame $3750-4150 \AA$ out of the full optical wavelength range available, $3800-9200 \AA$ in the observed frame. The eigencoefficients were used to correlate with physical properties of the galaxies. Later, Chen et al. (2012) estimated model-based stellar populations and dust parameters for a sample of SDSS galaxies. They used eigencoefficients calculated in the $3700-5500 \AA$ range, in conjunction with the Bayesian parameter estimation method.

Despite decades of effort and progress, the Lick/IDS indices remain subjective. The root cause lies in the difficulties in defining the true continua in the vicinity of a feature. Two main hurdles are: (1) there is no "true" continuum in late-type stars at the Lick/IDS spectral resolution and the continua are "pseudo" (Worthey et al. 1994), and (2) the location and the width of the pseudocontinua are subjective. The locations are chosen to flank 
the feature, and the width to be large enough in order to minimize the effect of typical stellar velocity dispersion of galaxies on the measured index strengths (Worthey et al. 1994; Trager et al. 1998). For a galaxy of a given velocity dispersion, these characteristics determine the absorption lines present within the pseudocontinua, and in turn impact the parameter sensitivity of the corresponding feature. For example, Jones \& Worthey (1995) found in a single-burst stellar population model that the narrow Balmer index, $\mathrm{H} \gamma_{\mathrm{HR}}$, is significantly more sensitive to stellar age than its broad counterpart, $\mathrm{H} \gamma_{A}$, suggesting that the broader Balmer indices may be contaminated by metal lines. They are the Fe I lines (Thomas et al. 2004), present in the pseudocontinua of the broad, higher-order Balmer indices (Worthey \& Ottaviani 1997).

The PCA approach (Wild et al. 2007; Chen et al. 2012) abandoned the use of pseudocontinua and bypassed difficulties (1) and (2), but the feature wavelength ranges were chosen subjectively to include the $4000 \AA$ break, unlike the Lick/IDS index system in which they were measured. As in pseudocontinua, the identity and the width of a feature can impact its parameter sensitivity, because they determine which absorption lines are present within. The reason for using PCA is that the eigenspectra are orthogonal, so that each eigenspectrum is potentially sensitive to individual physical parameters. Clearly, the next-generation approach is to combine the orthogonal-decomposition strength of PCA with objectivity in defining the feature wavelength regions.

The main goals of this paper are to identify the informative wavelength regions in an objective manner, and to associate physical significance with them. The analyses are performed on a solar-abundance model of simple stellar populations (SSPs) that is defined by two parameters, stellar age and metallicity. We use the CUR Matrix Decomposition (Drineas et al. 2008; Mahoney \& Drineas 2009), a powerful machine learning technique that has the ability to select statistically informative columns from a data matrix. The key characteristics of our approach are: (1) the continua are not explicitly used in the region identification, bypassing the abovementioned (1) and (2), which plague the Lick/IDS indices, and (2) different from the previous PCA approach (Wild et al. 2007; Chen et al. 2012), in this work the regions are determined objectively, in terms of both the identity and the width.

We cast an eye toward estimating physical parameters of real galaxies. This work results in two related applications. First, the identified regions and the parameter-eigencoefficient relation can be used to determine the stellar age and metallicity of those early-type galaxies that have solar abundance ratios, no dust, and a single-burst star formation history. Second, our region identification method can be applied to any set of model spectra. Users can generate their own parameter-eigencoefficient relation from the model, and use that to estimate parameters of galaxies from the observed spectra. Therefore, the intricate processes (Worthey \& Ottaviani 1997) involved in conforming to a common index system, which attempt to account for the difference in spectral resolution between the observed spectra and the system, can be eliminated.

In Section 2, we present the model spectra. In Section 3, we present the CUR Matrix Decomposition and describe how it is used to identify informative wavelength regions in the model spectra. In Section 4, we present the identified regions, the comparison to the existing line indices, and their physical significance. In Section 5, we conclude our work and discuss next steps. Vacuum wavelengths are used throughout the paper.

\section{MODEL AND PREPROCESSING}

We wish to derive a set of informative regions applicable for estimating the stellar age and metallicity in galaxies that have no dust and follow a single-burst star formation history. Hence, we perform the CUR Matrix Decomposition on a model with known physical parameters. The Bruzual \& Charlot (2003) stellar population model is used. For a chosen stellar initial mass function (IMF) and stellar isochrones, the model provides spectra of SSPs spanning six stellar metallicities by mass $(Z=0.0001,0.0004,0.004,0.008,0.02,0.05)$ and 221 stellar ages (0-20 Gyr, distributed roughly in equally sized logarithmic bins). We adopt the Chabrier (2003) IMF and Padova 1994 isochrones (Girardi et al. 1996 and references therein), and these choices are not expected to impact our results qualitatively. We do not add extinction to our model, and there is no stellar velocity dispersion variance in our model, in contrast to the galaxy spectra used in the Lick/IDS index measurement (Trager et al. 1998). We choose not to study the effect of noise and other artifacts on the identified regions, because other approaches (e.g., Robust PCA by Budavári et al. 2009) can be used to derive robust eigenspectra and eigencoefficients (Dobos et al. 2012) for regions in real galaxy spectra. The Bruzual \& Charlot (2003) model has solar abundance ratios, which may limit the application of our regions from many early-type galaxies (e.g., Worthey et al. 1992) with non-solar values. We decide to identify a new set of regions when a more sophisticated stellar population model is available in the future.

Preprocessing is carried out to ensure that (1) each and every spectrum is unique, ${ }^{6}$ (2) all of the flux values are available (i.e., no NaN, and no zero vector), and (3) the final ages and metallicities are selected so that they follow a rectangular parameter grid. This step results in the same six metallicities but 182 ages (2.5 Myr-20 Gyr), or 1092 SSP spectra in total.

The SSP are rebinned, in a flux-conserving fashion, to two configurations: the Lick/IDS and the SDSS. The Lick/IDS configuration allows us to compare the identified regions to the widely used indices in the literature. On the other hand, the SDSS configuration will result in regions with a spectral resolution higher than the Lick/IDS indices and closer to the SDSS galaxy spectra. The Lick/IDS configuration is a wavelength range $3800-6400 \AA$, a spectral resolution of $9 \AA$ FWHM, and a sampling of $1.25 \AA$ per pixel. The model spectra are therefore scaled down in resolution by convolving with a Gaussian function. The function has an FWHM equal to the Bruzual \& Charlot (2003) resolution ( $3 \AA$ FWHM within the optical) quadrature-subtracted from the targeted resolution. This choice is made to mimic the Lick/IDS spectrograph configuration (4000-6400 $\AA$ in air wavelengths; Burstein et al. 1984 ) as well as to include both the left and the right flanks of $\mathrm{D}_{n}(4000)$. They are referred as the $\mathrm{D}_{n}(4000) \mathrm{L}$ and $\mathrm{D}_{n}(4000) \mathrm{R}$ for convenience. The SDSS configuration is a wavelength range $3450-8350 \AA$, a spectral resolution of $3 \AA$ FWHM, and a sampling of $69 \mathrm{~km} \mathrm{~s}^{-1}$ per pixel. The choice of $3 \AA$ is limited by the instrumental resolution of the Bruzual \& Charlot (2003) model. The actual spectral resolution in the SDSS is 1800 (corresponds to $166 \mathrm{~km} \mathrm{~s}^{-1}$ ), higher than that of the model at the shortest wavelengths, and is $1.9 \AA$ at $\lambda=3450 \AA$. The wavelength range is tuned to coincide with the rest-frame

\footnotetext{
6 Some SSP spectra are found to be the same in the $\chi^{2}$ sense, despite the fact that they have different ages. For a given metallicity, we retain the spectrum at the earlier age when a pair of spectra at two consecutive ages are the same. The real degeneracies between age and metallicity still remain.
} 
Table 1

LeverageScoreSum of Extended Lick/IDS Indices, for Wavelength Range 3800-6400 ̊ at $9 \AA$ Spectral Resolution

\begin{tabular}{|c|c|c|c|c|c|c|}
\hline Region & Reference & Unit & $\lambda_{S}$ & $\lambda_{E}$ & $\lambda_{E}-\lambda_{S}$ & $\sum p_{\lambda}$ \\
\hline $\mathrm{D}_{n}(4000)$ & Balogh99 & $\mathrm{N} / \mathrm{A}$ & {$[3851.1,4001.1]$} & {$[3951.1,4101.2]$} & {$[100.0,100.0]$} & 0.199 \\
\hline $\mathrm{H} \beta$ & Trager98 & $\AA$ & 4849.2 & 4878.0 & 28.8 & 0.050 \\
\hline $\mathrm{H} \delta_{A}$ & WortheyOttaviani97 & $\AA$ & 4084.7 & 4123.4 & 38.8 & 0.040 \\
\hline G4300 & Trager98 & $\AA$ & 4282.6 & 4362.6 & 80.0 & 0.039 \\
\hline $\mathrm{C}_{2} 4668$ & Trager98 & $\AA$ & 4635.3 & 4721.6 & 86.3 & 0.027 \\
\hline $\mathrm{H} \delta_{F}$ & WortheyOttaviani97 & $\AA$ & 4092.2 & 4113.4 & 21.3 & 0.027 \\
\hline $\mathrm{H} \gamma_{A}$ & WortheyOttaviani97 & $\AA$ & 4321.0 & 4364.7 & 43.8 & 0.026 \\
\hline $\mathrm{TiO}_{2}$ & Trager98 & mag & 6191.3 & 6273.9 & 82.5 & 0.021 \\
\hline $\mathrm{Fe} 4531$ & Trager98 & $\AA$ & 4515.5 & 4560.5 & 45.0 & 0.021 \\
\hline Fe4383 & Trager98 & $\AA$ & 4370.4 & 4421.6 & 51.3 & 0.019 \\
\hline $\mathrm{H} \gamma_{F}$ & WortheyOttaviani97 & $\AA$ & 4332.5 & 4353.5 & 21.0 & 0.018 \\
\hline $\mathrm{Mg}_{2}$ & Trager98 & mag & 5155.6 & 5198.1 & 42.5 & 0.018 \\
\hline $\mathrm{CN}_{2}$ & Trager98 & mag & 4143.3 & 4178.3 & 35.0 & 0.018 \\
\hline $\mathrm{CN}_{1}$ & Trager98 & mag & 4143.3 & 4178.3 & 35.0 & 0.018 \\
\hline $\mathrm{Ca} 4455$ & Trager98 & $\AA$ & 4453.4 & 4475.9 & 22.5 & 0.018 \\
\hline Fe 5015 & Trager98 & $\AA$ & 4979.1 & 5055.4 & 76.3 & 0.016 \\
\hline $\mathrm{Mg}_{b}$ & Trager98 & $\AA$ & 5161.6 & 5194.1 & 32.5 & 0.016 \\
\hline $\mathrm{Na} D$ & Trager98 & $\AA$ & 5878.5 & 5911.0 & 32.5 & 0.012 \\
\hline $\mathrm{Mg}_{1}$ & Trager98 & mag & 5070.5 & 5135.6 & 65.0 & 0.011 \\
\hline $\mathrm{TiO}_{1}$ & Trager98 & mag & 5938.3 & 5995.8 & 57.5 & 0.009 \\
\hline $\mathrm{Fe} 5270$ & Trager98 & $\AA ̊$ & 5247.1 & 5287.1 & 40.0 & 0.008 \\
\hline Fe5406 & Trager98 & $\AA$ & 5389.0 & 5416.5 & 27.5 & 0.007 \\
\hline Fe5335 & Trager98 & $\AA$ & 5313.6 & 5353.6 & 40.0 & 0.005 \\
\hline $\mathrm{Ca} 4227$ & Trager98 & $\AA$ & 4223.4 & 4235.9 & 12.5 & 0.004 \\
\hline Fe5709 & Trager98 & $\AA$ & 5698.2 & 5722.0 & 23.8 & 0.004 \\
\hline Fe5782 & Trager98 & $\AA$ & 5778.2 & 5798.2 & 20.0 & 0.003 \\
\hline
\end{tabular}

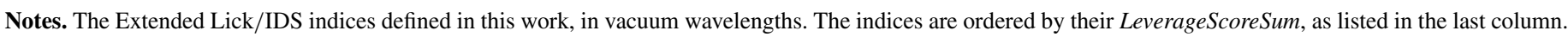
The $\lambda_{S}$ and $\lambda_{E}$ are the index wavelength bounds taken from the references.

wavelength range of spectra in most nearby SDSS galaxies, for which the median redshift is 0.1 . The observed-frame wavelengths of the SDSS spectrograph are ranged from 3800 to $9200 \AA$.

The spectra are converted from flux density (in $\operatorname{erg~s}^{-1} \AA^{-1}$ ) to flux (in erg s${ }^{-1}$ ) using $f_{\lambda}^{\prime}=f_{\lambda} \cdot \Delta \lambda$. The data values, which will be cast into a matrix later, are in the flux unit. The mean spectrum of the model is subtracted from each SSP spectrum. The data cloud formed by the spectra is hence centered in the wavelength space. The spectra are not continuum subtracted, and as such, no assumption on the true continuum is made.

\subsection{Extended Lick/IDS indices}

We assemble the 21 Lick/IDS indices from Trager et al. (1998, their whole Table 2), the four indices from Worthey \& Ottaviani (1997; $\mathrm{H} \delta_{A}, \mathrm{H} \gamma_{A}, \mathrm{H} \delta_{F}$, and $\mathrm{H} \gamma_{F}$, or their whole Table 1), and the 1 index from Balogh et al. (1999; $\mathrm{D}_{n}(4000)$ in Table 1), and name the 26 collectively "the Extended Lick/IDS indices" for convenience. The details are listed Table 1.

\section{ANALYSIS}

\subsection{CUR Matrix Decomposition}

The CUR Matrix Decomposition (Drineas et al. 2008; Mahoney \& Drineas 2009) is a novel method that has been used for large-scale data analysis in multiple disciplines. The main idea is to approximate a potentially huge data matrix with a lower rank matrix, where the latter is made with a small number of actual rows and/or columns of the original data matrix. The selected rows and columns are therefore statistically informative. The data in this work are model spectra; selecting the rows and columns corresponds to selecting the spectrum IDs and wavelengths, respectively. We will use only the column part of the decomposition, selecting informative wavelengths in the spectra. Further details of the CUR Matrix Decomposition are presented in Appendix A.

We start by constructing an $m \times n$ data matrix $A$, where each row is a single SSP spectrum from the model spectra. That is, $A_{i j}=f_{\lambda_{j}}^{i} \cdot \Delta \lambda_{j}$ is the flux of the $i$ th spectrum at the $j$ th wavelength. The full set of model spectra (Section 2) is used, so that the sample variance in the matrix is driven by both stellar age and metallicity. A singular value decomposition (SVD) is performed on $A$ :

$$
A=U \Sigma V^{T},
$$

which gives $U$ and $V$, the orthonormal matrices in which the columns are respectively the left and right singular vectors; and $\Sigma$, the diagonal matrix containing the singular values. For a chosen $k$, the LeverageScore at a given wavelength is

$$
p_{\lambda}=\frac{\sum_{j=1}^{k}\left(v_{\lambda}^{j}\right)^{2}}{k},
$$

where $v_{\lambda}^{j}$ is the $j$ th right singular vector. The collection of the $k$ right singular vectors forms an orthonormal basis to the fluxes in the high-dimensional wavelength space. The larger the $v_{\lambda}^{j}$ value, the larger the sample variance is projected from the $j$ th basis vector onto the given wavelength axis. The $p_{\lambda}$ is hence proportional to the total projected sample variance from altogether $k$ basis vectors onto that wavelength axis. In other words, $p_{\lambda}$ measures the information contained in a wavelength. This information tells the sample variance driven by the physical parameters stellar age and metallicity. 


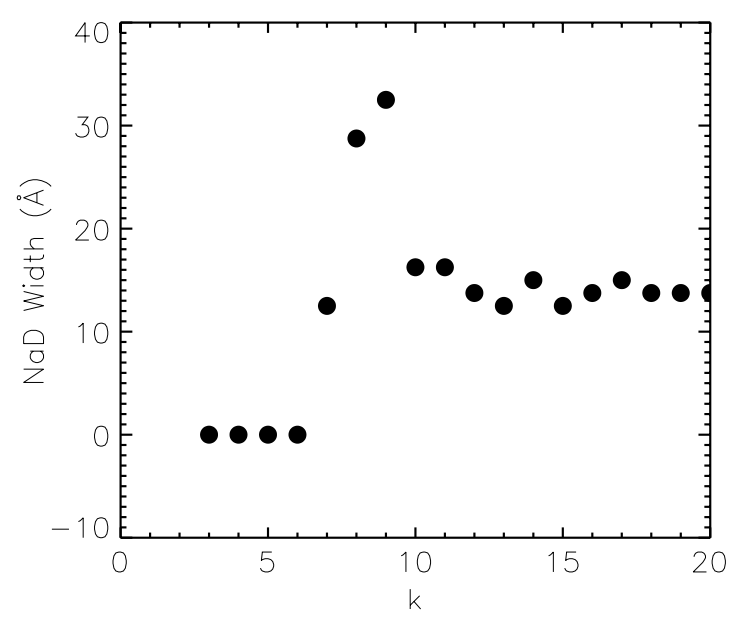

Figure 1. Width of the $\mathrm{Na} \mathrm{D}$ region as a function of $k$, for $T=0.7$ in the Lick/IDS configuration. The width converges.

The ordering of the spectra per row in the matrix $A$ does not impact the ordering of the right singular vectors. As long as the same set of spectra is used, and the same $k$ is chosen, the same $p_{\lambda}$ will result. The $k$ values will be chosen in an objective manner in this work.

\subsection{Region Identification Procedure}

The two main steps in the region identification are to select the informative wavelength, $\lambda_{I}$, and to determine its region of influence, ROI. To select $\lambda_{I}$, we pick from the available wavelengths the one with the highest $p_{\lambda}$. The procedure for ROI determination is developed based on several considerations: (1) a region is contiguous in wavelength, (2) a region is allowed to be asymmetric about $\lambda_{I}$, (3) regions can overlap with each other, and (4) we expect that, if a region can indeed be defined, the $p_{\lambda}$ would decline to zero when approaching the left and right wavelength bounds, because the fluxes. Combining the above considerations, an ROI is calculated as follows. Starting at the wavelength that is selected $\left(\lambda_{I}\right)$, we attempt to include a pixel to its immediate left or right. The accumulated $p_{\lambda}$ of both scenarios are calculated, called $p_{\lambda}^{S}$. The pixel that gives the higher $p_{\lambda}^{S}$ is added to the region. The procedure is repeated until the last (ith) pixel is included, such that the following convergence criterion is satisfied:

$$
\left|\frac{p_{\lambda i}^{S}-p_{\lambda i-1}^{S}}{\lambda_{i}-\lambda_{i-1}}\right| \leqslant T \cdot \frac{\sum_{\lambda=\lambda_{1}}^{\lambda_{N}} p_{\lambda}}{\lambda_{N}-\lambda_{1}}=T \cdot \frac{1}{\lambda_{N}-\lambda_{1}} .
$$

That is, the change of the information content of a region per unit wavelength is a constant. We tie the constant to the average information expected in the case where every wavelength is equally informative. The threshold, $T$, is set to be 0.7 in this work. This value is not unity, consistent with the fact that the $p_{\lambda}$ are not uniform. More importantly, this choice enables us to obtain converged Na D-region width (Section 4) for both the Lick/IDS and the SDSS configurations (Figures 1 and 2, respectively). The atomic lines, such as $\mathrm{Na} \mathrm{D}$, have welldefined central wavelengths and widths. They are therefore good standards for checking various identification approaches. $T=0.7 \pm 0.1$ also give the width convergence in the SDSS configuration. The generality of this convergence for arbitrary spectra is however not yet established.

An ROI is fully indicated by the left and right wavelength bounds $\left[\lambda_{S}, \lambda_{E}\right]$. Once the $\lambda_{I}$ and the $\left[\lambda_{S}, \lambda_{E}\right]$ are determined,

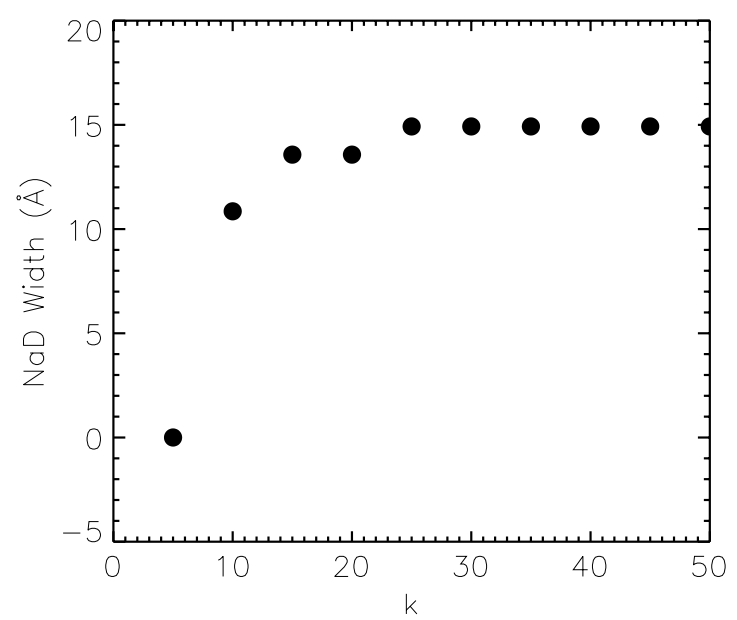

Figure 2. Width of the $\mathrm{Na} \mathrm{D}$ region as a function of $k$, for $T=0.7$ in the SDSS configuration. The width converges.

they are labeled as a "Region" and all of the involved pixels are masked out in the next $\lambda_{I}$ and ROI selection. The process is repeated until either no more pixels are available, or the desired number of regions is reached. We then use

$$
\text { LeverageScoreSum }=p^{S}=\sum_{\lambda=\lambda_{S}}^{\lambda_{E}} p_{\lambda}
$$

as the measure for the information contained in a region.

\subsection{Relation of CUR to PCA}

PCA has become a standard technique in spectral analyses. In extragalactic studies, it has been applied to remove sky from galaxy spectra (Wild \& Hewett 2005), to understand the diversity in galaxies (e.g., Connolly et al. 1995; Yip et al. 2004a; Dobos et al. 2012) and quasars (e.g., Francis et al. 1992; Shang et al. 2003; Yip et al. 2004b), to separate host galaxy contribution from broadline active galactic nucleus spectra (Vanden Berk et al. 2006), and to find supernovae in large galaxy spectral samples (Madgwick et al. 2003; Krughoff et al. 2011, and many more studies). These works used the fact that PCA compresses the data in the object space, leaving the number of wavelength bins in each eigenspectrum unchanged from that of the input spectra. The CUR Matrix Decomposition provides us a new way to compress the data, so that even the variable space can be compressed. The variable in the current context is the wavelength.

\subsection{Spectrum Cutout Analysis}

The next step is to compare the regions quantitatively. We prepare spectral segments of each region that are cut out from the model spectra. The set of cutouts of each region form a column subspace (in $\mathbb{R}^{m}$ ) of the original $m \times n$ data matrix $A$ (e.g., Strang 1988). The number of cutout pixels is less than the number of spectra in this work. Therefore, the rank of the subspace, at maximum, is equal to the number of pixels of that region. Its actual value would depend on the dimension of the subspace in question. Three subspace measures are used: (1) the cosine of the angle between two subspaces as defined by Gunawan et al. (2005), $\cos \theta_{\mathrm{GNS}}$; (2) the dot product between the first left-singular vectors of the two subspaces, $\cos \theta_{\mathrm{PC} 1}$; and 
Table 2

Informative Wavelength Regions, for Wavelength Range 3800-6400 A at $9 \AA$ Spectral Resolution

\begin{tabular}{|c|c|c|c|c|c|c|c|c|c|c|c|}
\hline Region & $\lambda_{S}$ & $\lambda_{I}$ & $\lambda_{E}$ & $\lambda_{E}-\lambda_{S}$ & $\lambda_{I}-\lambda_{S}$ & $\sum p_{\lambda}$ & Lick/IDS Overlaps & First Mode & $u^{1} \cdot p$ & Second Mode & $u^{2} \cdot p$ \\
\hline Region01 & 3801.2 & 3932.5 & 4053.8 & 252.5 & 131.2 & 0.312 & $\mathrm{D}_{n}(4000) \mathrm{L} ; \mathrm{D}_{n}(4000) \mathrm{R}$ & AGE & -0.395 & METAL & -0.135 \\
\hline Region02 & 4761.2 & 4856.2 & 4908.8 & 147.5 & 95.0 & 0.104 & $\mathrm{H} \beta$ & AGE & -0.393 & METAL & -0.163 \\
\hline Region03 & 4080.0 & 4101.2 & 4183.8 & 103.8 & 21.2 & 0.075 & $\mathrm{CN}_{1} ; \mathrm{CN}_{2} ; \mathrm{H} \delta_{A} ; \mathrm{H} \delta_{F} ; \mathrm{D}_{n}(4000) \mathrm{R}$ & AGE & -0.396 & AGE & 0.208 \\
\hline Region12 & 4497.5 & 4505.0 & 4548.8 & 51.2 & 7.5 & 0.028 & $\mathrm{Fe} 4531$ & AGE & -0.397 & AGE & 0.054 \\
\hline Region05 & 4321.2 & 4340.0 & 4351.2 & 30.0 & 18.8 & 0.022 & $\mathrm{G} 4300 ; \mathrm{H} \gamma_{A} ; \mathrm{H} \gamma_{F}$ & AGE & -0.392 & AGE & 0.120 \\
\hline Region04 & 4461.2 & 4470.0 & 4486.2 & 25.0 & 8.8 & 0.020 & $\mathrm{Ca} 4455$ & AGE & -0.398 & METAL & -0.277 \\
\hline Region06 & 5162.5 & 5167.5 & 5188.8 & 26.2 & 5.0 & 0.015 & $\mathrm{Mg}_{2} ; \mathrm{Mg}_{b}$ & AGE & -0.388 & METAL & 0.166 \\
\hline Region08 & 4636.2 & 4648.8 & 4652.5 & 16.2 & 12.5 & 0.010 & $\mathrm{C}_{2} 4668$ & AGE & -0.394 & METAL & -0.274 \\
\hline Region11 & 5885.0 & 5891.2 & 5901.2 & 16.2 & 6.2 & 0.008 & $\mathrm{Na} \mathrm{D}$ & AGE & -0.374 & AGE & 0.073 \\
\hline Region13 & 4061.2 & 4071.2 & 4078.8 & 17.5 & 10.0 & 0.008 & $\mathrm{D}_{n}(4000) \mathrm{R}$ & AGE & -0.398 & METAL & -0.333 \\
\hline Region10 & 4191.2 & 4198.8 & 4202.5 & 11.2 & 7.5 & 0.007 & $\cdots$ & AGE & -0.397 & METAL & 0.263 \\
\hline Region15 & 4292.5 & 4300.0 & 4308.8 & 16.2 & 7.5 & 0.007 & G4300 & AGE & -0.398 & METAL & 0.155 \\
\hline Region16 & 4257.5 & 4266.2 & 4273.8 & 16.2 & 8.8 & 0.007 & $\ldots$ & AGE & -0.397 & METAL & -0.140 \\
\hline Region09 & 4381.2 & 4387.5 & 4391.2 & 10.0 & 6.2 & 0.007 & $\mathrm{Fe} 4383$ & AGE & -0.398 & METAL & -0.174 \\
\hline Region07 & 4913.8 & 4921.2 & 4923.8 & 10.0 & 7.5 & 0.006 & $\ldots$ & AGE & -0.393 & METAL & -0.349 \\
\hline Region17 & 5262.5 & 5265.0 & 5272.5 & 10.0 & 2.5 & 0.005 & Fe5270 & AGE & -0.388 & METAL & -0.043 \\
\hline Region 20 & 5872.5 & 5876.2 & 5883.8 & 11.2 & 3.8 & 0.004 & $\mathrm{Na} \mathrm{D}$ & AGE & -0.375 & METAL & 0.244 \\
\hline Region14 & 4411.2 & 4415.0 & 4420.0 & 8.8 & 3.8 & 0.004 & $\mathrm{Fe} 4383$ & AGE & -0.399 & AGE & -0.097 \\
\hline Region18 & 4311.2 & 4315.0 & 4317.5 & 6.2 & 3.8 & 0.003 & G4300 & AGE & -0.398 & METAL & -0.154 \\
\hline Region19 & 4393.8 & 4395.0 & 4398.8 & 5.0 & 1.2 & 0.002 & $\mathrm{Fe} 4383$ & AGE & -0.399 & METAL & -0.139 \\
\hline
\end{tabular}

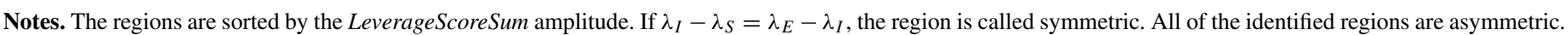

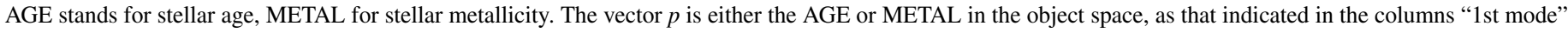

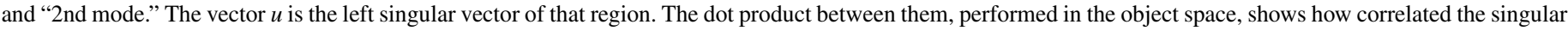

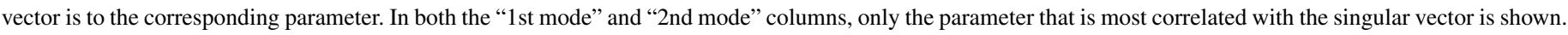
The extra horizontal line divides the regions with LeverageScoreSum less than and larger than 0.03.

(3) the Pearson correlation coefficient between the integrated fluxes of the two regions, ${ }^{7} r$.

The subspace measure (1) is calculated as follows. For the $i$ th region with number of wavelength bins equal to $n^{(i)}$, we cut out from the original data matrix $A$ the corresponding submatrix $A^{(i)}$, size $m \times n^{(i)}$. An SVD is performed on the submatrix, $A^{(i)}=U^{(i)} \Sigma^{(i)} V^{(i)^{T}}$. The angle between the $i$ th and the $j$ th regions, with $1 \leqslant n^{(i)} \leqslant n^{(j)}$, is calculated by applying the formula in Gunawan et al. (2005):

$$
\cos \theta_{\mathrm{GNS}}=\sqrt{\operatorname{det}\left(M^{T} M\right)},
$$

where we put the matrix $M=U^{(j)^{T}} U^{(i)}$. The symbol "det" denotes the determinant, and $T$ the transpose, of a matrix. They have shown that $\cos \theta_{\mathrm{GNS}}$ is proportional to the volume of the parallelepiped spanned by the projection of the basis vectors of the lower-dimensional subspace on the higher-dimensional subspace. The one-dimensional (1D) case is helpful for us to grasp the picture: the matrix product $M^{T} M$ becomes the dot product between the two 1D basis vectors.

From the SVD of the regions we can also calculate the dot product between the first left-singular vectors of any two regions, i.e., the subspace measure (2). Indeed, any order of singular vector can be considered, but we pick the first vector because it captures the maximum sample variance.

\subsection{Parameter Sensitivity of Regions}

To associate physical significance with the regions, we explore the correlation between a chosen parameterization of

\footnotetext{
7 While the subspaces spanned by the cutouts - the spectra of narrow wavelength range - are expected to be fairly linear individually, correlation among the subspaces may not be the case. In this work (Section 4.4,

Figure 12), we will show a posteriori that the Pearson correlation coefficient, a good measure for linear correlation, is unlikely to be the best for probing the relation among regions.
}

a region and the physical parameters defining the model. We start by examining the Pearson correlation coefficient formula ${ }^{8}$; the correlation between two variables $X$ and $Y$ (each has $m$ components, $m$ being the number of spectra/objects) is equivalent to the dot product between two vectors in the object space (each vector has $m$ components). We hereafter use "the correlation between two subspaces (in the variable space)" and "the angle between two subspaces (in the object space)" interchangeably.

This rather simple observation is highly instrumental for quantifying the parameter sensitivity of a region. If the subspace formed by a region is correlated with a parameter, then the two vectors-one is a chosen parameterization of the subspace, and another is the parameter itself (vector $p$ ); both have $m$ components-should be parallel to each other in the object space. The vector $p$ is either stellar age or metallicity. The angle between the two vectors can be represented by their dot product. We pick two parameterizations for a region, namely, the first and the second left-singular vectors (vectors $u^{1}$ and $u^{2}$ ), as most of the information is encapsulated by the lowest-order modes.

\section{RESULTS}

\subsection{Informative Regions: Lick/IDS Configuration}

The identified wavelength regions using our approach are shown in Figure 3 . The corresponding $\lambda_{I}, \lambda_{S}, \lambda_{E}$, as well as the overlapping Extended Lick/IDS indices, are given in Table 2. From the width convergence of the $\mathrm{Na} \mathrm{D}$ region (Figure 4), $k$ is set to 10 . The following regions are found, in the order of importance.

\footnotetext{
8 Here we examine the Pearson correlation coefficient from a general perspective, in a different context from Section 3.4.
} 


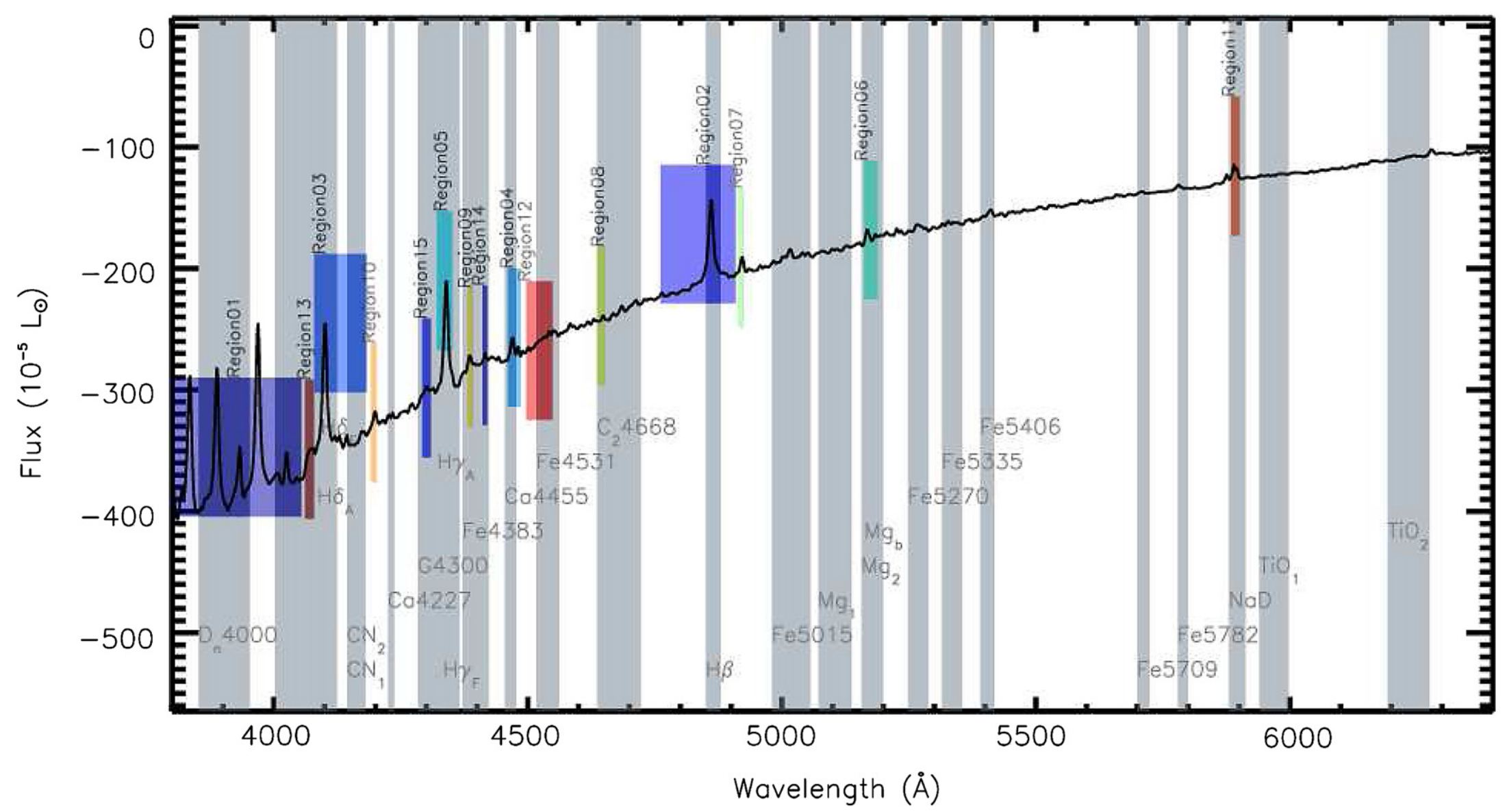

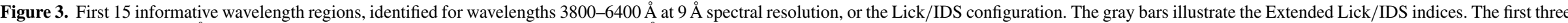

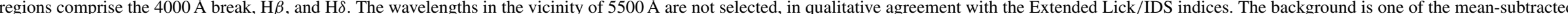
spectra in the model. The bottom of a region label marks the $\lambda_{I}$ of that region.

(A color version of this figure is available in the online journal.) 


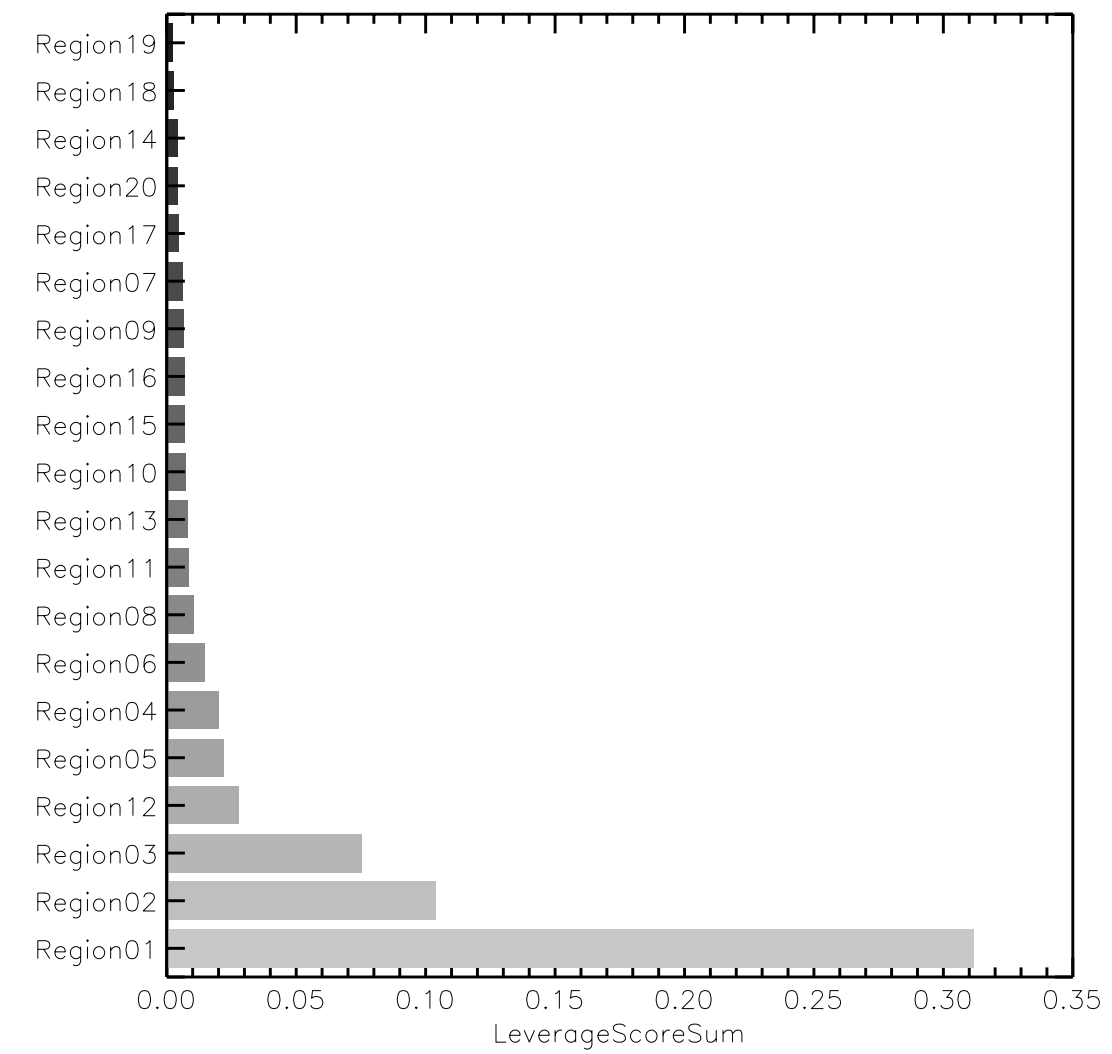

Figure 4. LeverageScoreSum of the identified regions in the Lick/IDS configuration, sorted by amplitude. The first three regions are most informative.

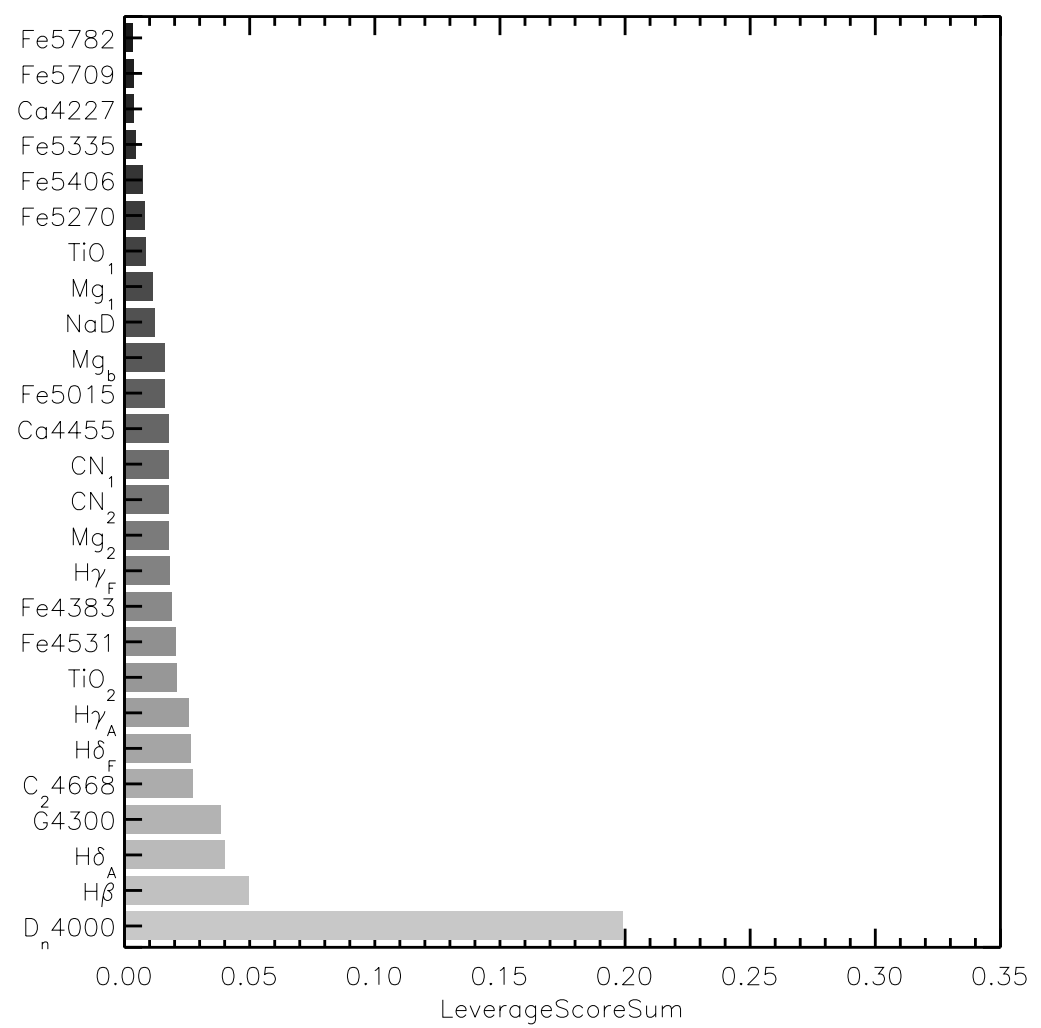

Figure 5. LeverageScoreSum of the Extended Lick/IDS indices, sorted by amplitude. We note that there was no importance ordering in the Extended Lick/IDS indices originally. The indices $\mathrm{D}_{n}(4000), \mathrm{H} \beta$, and $\mathrm{H} \delta_{A}$ are found to be most informative.

1. 1st. Comprises the $4000 \AA$ A break. The LeverageScoreSum is a factor of a few or more larger than those of the other regions.

2. 2nd. Comprises the $\mathrm{H} \beta$ line.
3. 3rd. Comprises the $\mathrm{H} \delta$ line. The LeverageScoreSum is comparable to that of the $\mathrm{H} \beta$ region.

4. 4th. Comprises the Fe $\lambda 4531$ line, which belongs to the "Fe-like indices" family (Trager et al. 1998). 
Table 3

Informative Wavelength Regions, for Wavelength Range 3450-8350 Å at $3 \AA$ Spectral Resolution

\begin{tabular}{|c|c|c|c|c|c|c|c|c|c|c|c|c|}
\hline Region & $\lambda_{S}$ & $\lambda_{I}$ & $\lambda_{E}$ & $\lambda_{E}-\lambda_{S}$ & $\lambda_{I}-\lambda_{S}$ & $\sum p_{\lambda}$ & Lick/IDS Overlaps & First Mode & $\bar{~} u^{1} \cdot p$ & Second Mode & $\overline{u u^{2} \cdot p}$ & Optical Line Overlaps \\
\hline Region01 & 3450.8 & 3934.5 & 4125.6 & 674.8 & 483.8 & 0.403 & $\mathrm{H} \delta_{A} ; \mathrm{H} \delta_{F} ; \mathrm{D}_{n}(4000) \mathrm{L} ; \mathrm{D}_{n}(4000) \mathrm{R}$ & AGE & -0.390 & METAL & -0.079 & $\begin{array}{c}\mathrm{H} \theta \lambda 3799 ; \mathrm{H} \eta \lambda 3836 ; \mathrm{H} \zeta \lambda 3890 ; \\
\mathrm{CaK} \lambda 3935 ; \mathrm{CaH} \lambda 3970 ; \mathrm{H} \epsilon \lambda 3971 ; \\
\mathrm{H} \delta \lambda 4103\end{array}$ \\
\hline Region08 & 4378.0 & 4472.7 & 4574.7 & 196.8 & 94.7 & 0.063 & $\mathrm{Fe} 4383 ; \mathrm{Ca} 4455 ; \mathrm{Fe} 4531$ & AGE & -0.397 & METAL & -0.137 & $\ldots$ \\
\hline Region03 & 4773.7 & 4861.3 & 4899.5 & 125.8 & 87.6 & 0.056 & $\mathrm{H} \beta$ & AGE & -0.393 & METAL & -0.171 & $\mathrm{H} \beta \lambda 4863$ \\
\hline Region04 & 4238.2 & 4340.9 & 4377.0 & 138.8 & 102.7 & 0.049 & $\mathrm{G} 4300 ; \mathrm{Fe} 4383 ; \mathrm{H} \gamma_{A} ; \mathrm{H} \gamma_{F}$ & AGE & -0.396 & METAL & -0.177 & $\mathrm{H} \gamma \lambda 4342$ \\
\hline Region14 & 4135.1 & 4144.6 & 4237.2 & 102.1 & 9.5 & 0.028 & $\mathrm{CN}_{1} ; \mathrm{CN}_{2} ; \mathrm{Ca} 4227$ & AGE & -0.398 & METAL & -0.220 & .. \\
\hline Region12 & 4594.8 & 4651.2 & 4656.5 & 61.7 & 56.4 & 0.015 & $\mathrm{C}_{2} 4668$ & AGE & -0.395 & METAL & -0.255 & $\ldots$ \\
\hline Region02 & 6549.4 & 6564.5 & 6576.6 & 27.2 & 15.1 & 0.012 & $\ldots$ & AGE & -0.371 & METAL & 0.253 & $\mathrm{H} \alpha \lambda 6565$ \\
\hline Region09 & 8219.7 & 8229.2 & 8259.5 & 39.8 & 9.5 & 0.009 & .. & AGE & -0.370 & METAL & 0.290 & $\ldots$ \\
\hline Region 10 & 7166.1 & 7187.6 & 7197.5 & 31.4 & 21.5 & 0.008 & $\cdots$ & AGE & -0.375 & METAL & 0.305 & $\cdots$ \\
\hline Region06 & 5165.9 & 5168.2 & 5179.0 & 13.1 & 2.4 & 0.006 & $\mathrm{Mg}_{2} ; \mathrm{Mg}_{b}$ & AGE & -0.388 & METAL & 0.157 & $\operatorname{Mg}_{I} \lambda 5169 ; \operatorname{Mg}_{1} \lambda 5174$ \\
\hline Region11 & 5887.3 & 5892.8 & 5902.3 & 14.9 & 5.4 & 0.006 & $\mathrm{NaD}$ & AGE & -0.374 & METAL & 0.093 & Na I $\lambda 5892 ; \mathrm{Na}$ I $\lambda 5898$ \\
\hline Region19 & 4914.2 & 4924.4 & 4936.9 & 22.7 & 10.2 & 0.006 & $\cdots$ & AGE & -0.393 & METAL & -0.388 & $\cdots$ \\
\hline Region18 & 8159.4 & 8178.2 & 8182.0 & 22.6 & 18.8 & 0.005 & $\cdots$ & AGE & -0.373 & METAL & 0.301 & $\cdots$ \\
\hline Region17 & 4681.3 & 4686.6 & 4694.2 & 12.9 & 5.4 & 0.004 & $\mathrm{C}_{2} 4668$ & AGE & -0.393 & AGE & 0.035 & $\cdots$ \\
\hline Region07 & 4712.6 & 4714.8 & 4720.2 & 7.6 & 2.2 & 0.003 & $\mathrm{C}_{2} 4668$ & AGE & -0.393 & METAL & 0.428 & $\cdots$ \\
\hline Region20 & 5180.2 & 5183.7 & 5189.7 & 9.5 & 3.6 & 0.003 & $\mathrm{Mg}_{2} ; \mathrm{Mg}_{b}$ & AGE & -0.387 & METAL & 0.164 & $\cdots$ \\
\hline Region13 & 5224.5 & 5226.9 & 5231.7 & 7.2 & 2.4 & 0.003 & $\ldots$ & AGE & -0.387 & METAL & 0.121 & $\ldots$ \\
\hline Region05 & 8090.2 & 8092.1 & 8097.7 & 7.5 & 1.9 & 0.003 & $\ldots$ & AGE & -0.364 & METAL & -0.121 & $\ldots$ \\
\hline Region16 & 7274.1 & 7277.4 & 7279.1 & 5.0 & 3.3 & 0.002 & $\ldots$ & AGE & -0.375 & METAL & 0.335 & $\ldots$ \\
\hline Region15 & 5053.0 & 5054.1 & 5056.5 & 3.5 & 1.2 & 0.002 & Fe5015 & AGE & -0.391 & METAL & -0.143 & $\ldots$ \\
\hline
\end{tabular}

Notes. See footnote in Table 2. This table lists the Lick/IDS indices, and the prominent optical absorption lines, which overlap with the identified regions. 


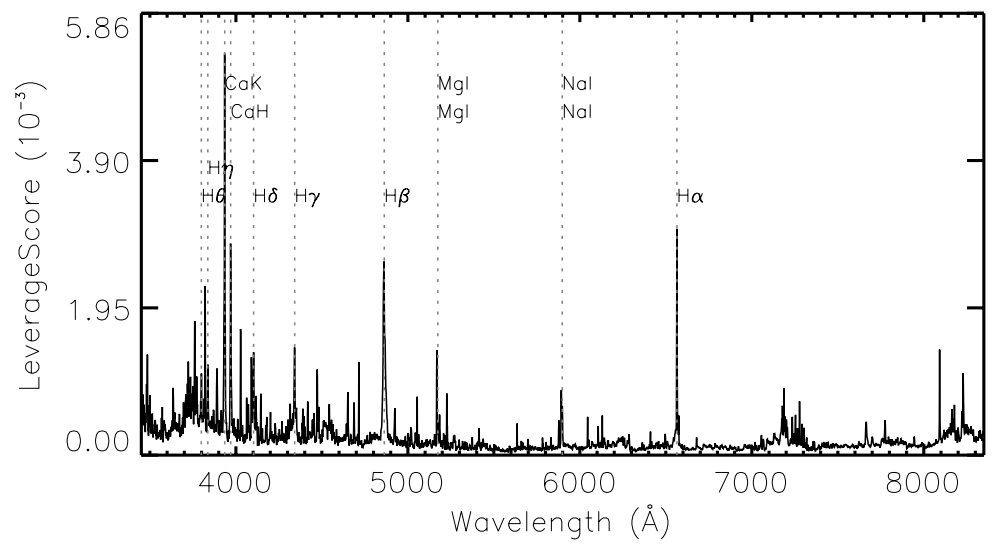

Figure 6. LeverageScore measures the information contained in a wavelength of a set of spectra in the SDSS configuration. Some absorption lines are marked.

5. 5th. Comprises the $G$ band. The $G$ band is primarily arisen from $\mathrm{CH}$ molecules and their energy levels are therefore many. Not too surprisingly, it is blended with $\mathrm{H} \gamma$ to form a single region. The $G$ band and the $\mathrm{H} \gamma$ indices are also not disjoint in wavelength in the Extended Lick/IDS indices definition (Table 1).

6. Higher orders. Certain Extended Lick/IDS indices are recovered in the higher-order regions.

The LeverageScoreSum of our indices are plotted in Figure 4. The first three regions are most informative. As a comparison, we also calculate the LeverageScoreSum of the Extended Lick/IDS indices, shown in Figure 5. There was no importance ordering in the Extended Lick/IDS indices originally but they are sorted here nonetheless. The widely used indices, $\mathrm{D}_{n}(4000), \mathrm{H} \beta$, and $\mathrm{H} \delta_{A}$, are found indeed to be most informative.

We also see that the wavelengths in the vicinity of $5500 \AA$ are not selected, in qualitative agreement with the Extended Lick/IDS indices (Figure 3). The wavelengths may be insensitive to stellar age or stellar metallicity.

\subsection{Informative Regions: SDSS Configuration}

To identify informative regions in the SDSS configuration, the first step is to determine the appropriate $k$ value. Because of the higher resolution, the rank of the data matrix $A$ can be larger than the Lick/IDS case and the appropriate $k$ value can be different. Examine again the $\mathrm{Na} \mathrm{D}$ region width as a function of $k$ (Figure 2); it converges to $14.9 \AA$ with increasing $k$. We use $k=$ 25 in the region identification. The LeverageScore as a function of wavelength is plotted in Figure 6, with high amplitude seen in some absorption lines, and in the vicinity of the $4000 \AA$ break. The most informative regions are plotted in Figure 7. They are as follows, in the order of importance.

1. 1st. Comprises the $4000 \AA$ break and the $\mathrm{H} \delta$ line. In the Extended Lick/IDS indices, as we noted earlier, the $\mathrm{D}_{n}(4000) \mathrm{R}$ and $\mathrm{H} \delta_{A}$ overlap in wavelength. So it is not entirely surprising that they form a single region. For example, there are likely many metal lines present between the $\mathrm{D}_{n}(4000) \mathrm{L}$ and $\mathrm{D}_{n}(4000) \mathrm{R}$, so that $\mathrm{D}_{n}(4000) \mathrm{L}, \mathrm{D}_{n}(4000) \mathrm{R}$, and the $\mathrm{H} \delta$ form one single region.

2. 2nd. Comprises the Fe-like indices(Trager et al. 1998). This region appears to be more sensitive to stellar metallicity than the most informative region (the $4000 \AA$ break and the $\mathrm{H} \delta$ line), concluded from the dot product between the 2nd left-singular and stellar metallicity vectors (the last column of Table 3). Unfortunately, the LeverageScoreSum is a factor of six smaller, meaning this region is less informative. We have yet to connect the two different quantities- the LeverageScoreSum and the dot product-to form a single measure for parameter sensitivity, which is beyond the scope of this work.

3. $3 r d$. Comprises the $\mathrm{H} \beta$ line.

4. 4th. Comprises the $G$ band and the $\mathrm{H} \gamma$ line. The LeverageScoreSum amplitudes are comparable in the 2nd, 3rd, and 4th most informative regions, about 0.05 .

5. Higher orders. The 7th most informative region comprises the $\mathrm{H} \alpha$ (Table 3), which is detectable in most SDSS galaxies but falls outside of the Lick/IDS spectrograph wavelength range.

The comparison of the regions between the Lick/IDS and the SDSS configurations is illustrated in Figure 8. For both sets, the $4000 \AA$ break, $\mathrm{H} \beta$, and $\mathrm{H} \delta$ regions are among the top three most informative. However, we expect the identified regions to depend on the spectral resolution and the wavelength coverage. To demonstrate the wavelength-coverage dependence, an extra region identification is carried out where we use the Lick/IDS spectral resolution and pixel size but the SDSS wavelength coverage (not shown). While the $4000 \AA$ break remains the most informative region identified, its ROI is different from that obtained in the Lick/IDS configuration, and is more similar to that obtained in the SDSS configuration. One possibility is that the true ROI of the $4000 \AA$ break exceeds the shortest wavelength of the Lick/IDS configuration.

The dependence of the regions on the spectral resolution is difficult to be quantified generally. When the resolution is low, blended features cannot be resolved. The identified region can become broader than the true wavelength bound, where the latter may be identifiable only in higher-resolution spectra. When the resolution is high, the identified regions may not be optimal in terms of studying lower-resolution spectra, or they may not be state-of-the-art for future surveys. To avoid these complicated situations, it is desirable to treat the identification method, instead of the identified regions, as being general. As such, the method can be applied to any set of spectra of the user's interest.

\subsection{Relation Among Regions}

The angle between the subspaces spanned by any two regions is shown in Figure 9, in $\cos ^{2} \theta_{\text {GNS }}$ (Gunawan et al. 2005). Those for the Extended Lick/IDS indices are shown in Figure 10. All of the higher-order regions are correlated with the first region, 


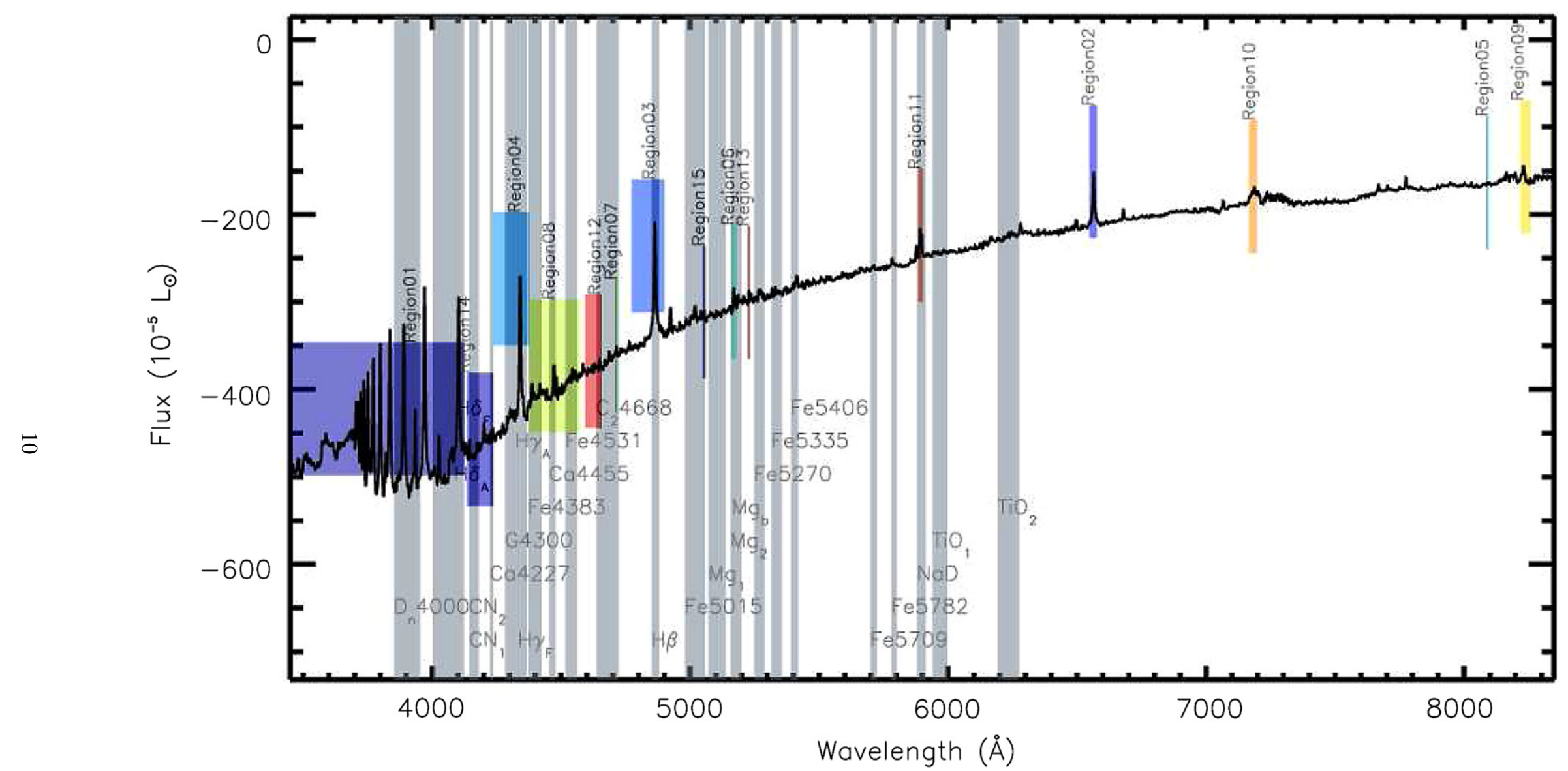

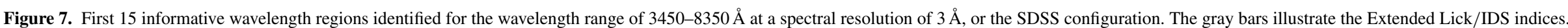

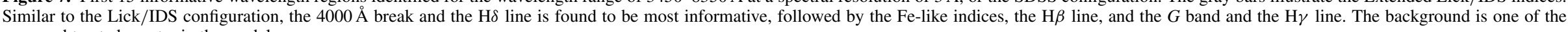
mean-subtracted spectra in the model.

(A color version of this figure is available in the online journal.) 


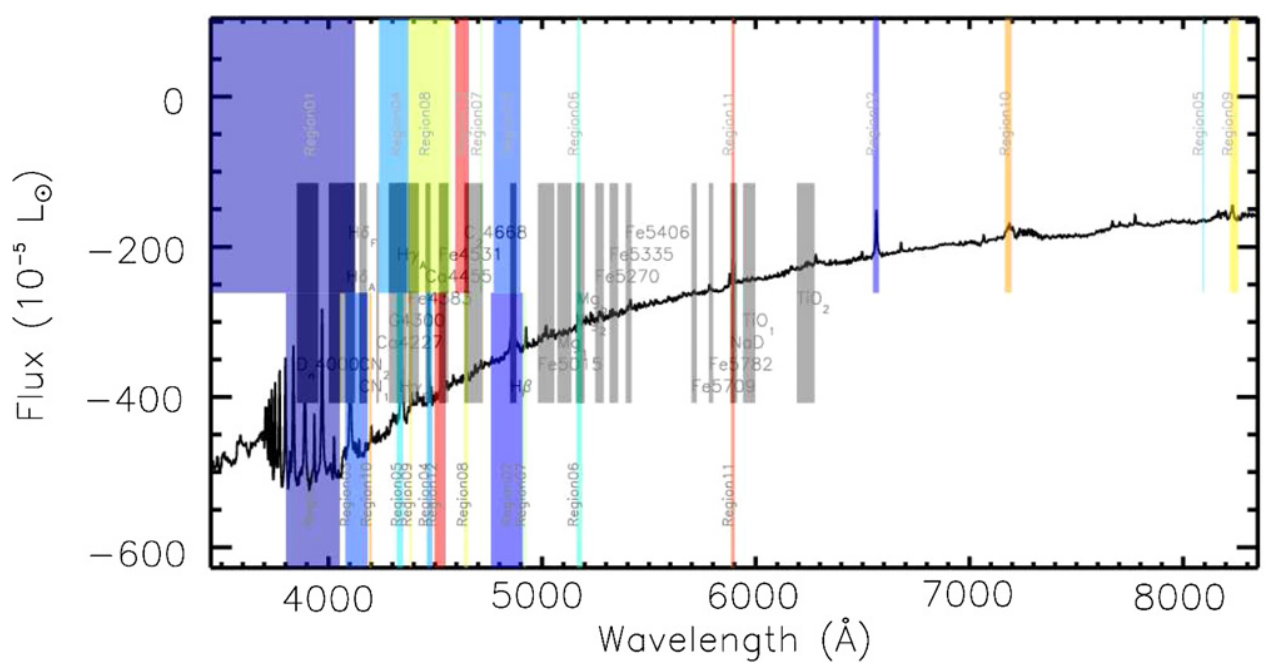

Figure 8. Comparison of the first 12 informative regions that are identified in the Lick/IDS (bottom) and SDSS (top) configurations. The gray bars show the Extended Lick/IDS indices.

(A color version of this figure is available in the online journal.)
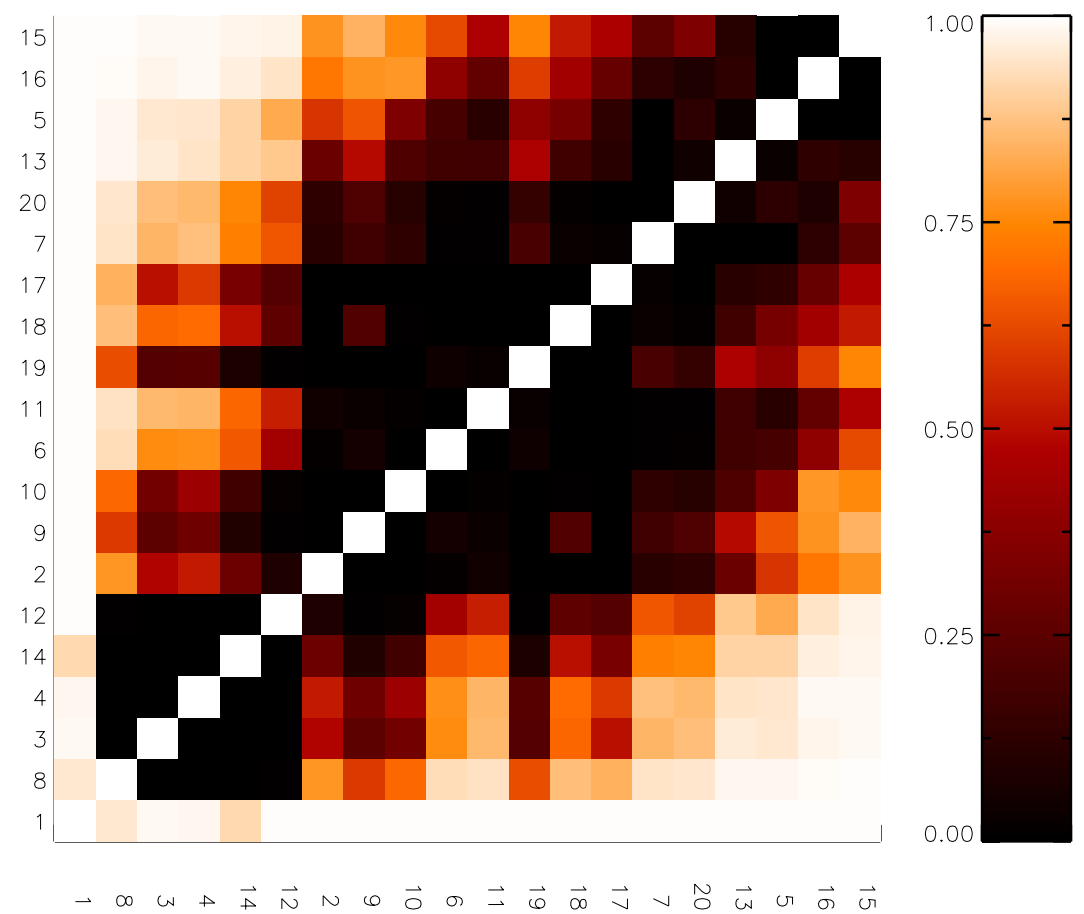

Figure 9. $\cos ^{2} \theta_{\mathrm{GNS}}$ between the subspaces spanned by the spectrum cutouts of our identified regions in the SDSS configuration. The line indices are sorted by their LeverageScoreSum. All regions are almost parallel to Region01.

(A color version of this figure is available in the online journal.)

suggesting that the higher-order regions are not substantially different. This is not surprising considering the SSPs are defined by two parameters only, namely the stellar age and metallicity, and that the $4000 \AA$ break is sensitive to both parameters. Some of the Extended Lick/IDS indices are also correlated with the $\mathrm{D}_{n}(4000)$ index, but not as many as in the Region01, or as high as the correlation amplitudes. This result shows that Region01 is more "complete" than the $\mathrm{D}_{n}(4000)$, in the sense that the corresponding subspace encapsulates most of the data directions.

The other pronounced difference between the Extended Lick/IDS indices and the regions is that, in the former, there are many more cases where two indices are orthogonal. The region representation is therefore more "compact," in the sense that we need fewer regions to encapsulate the various data directions.

The squared ${ }^{9}$ Pearson correlation coefficient $\left(r^{2}\right)$ between the integrated flux of two regions, and the squared dot product between the first left-singular vectors of two subspaces $\left(\cos ^{2} \theta_{\mathrm{PC} 1}\right)$, are shown side by side in Figure 11. A large correlation amplitude is seen between most region pairs. Using the integrated flux or the first singular vector to represent a subspace conveys less information about that subspace than using a number of

\footnotetext{
9 The squared Pearson correlation coefficient $\left(r^{2}\right)$ is used because we would like to focus on the amplitude of the correlation. It ranges from 0 to 1,0 if no correlation, 1 if $100 \%$ correlation. A consequence is the same color coding in the $r^{2}$ and $\cos ^{2} \theta_{\mathrm{GNS}}$ figures.
} 


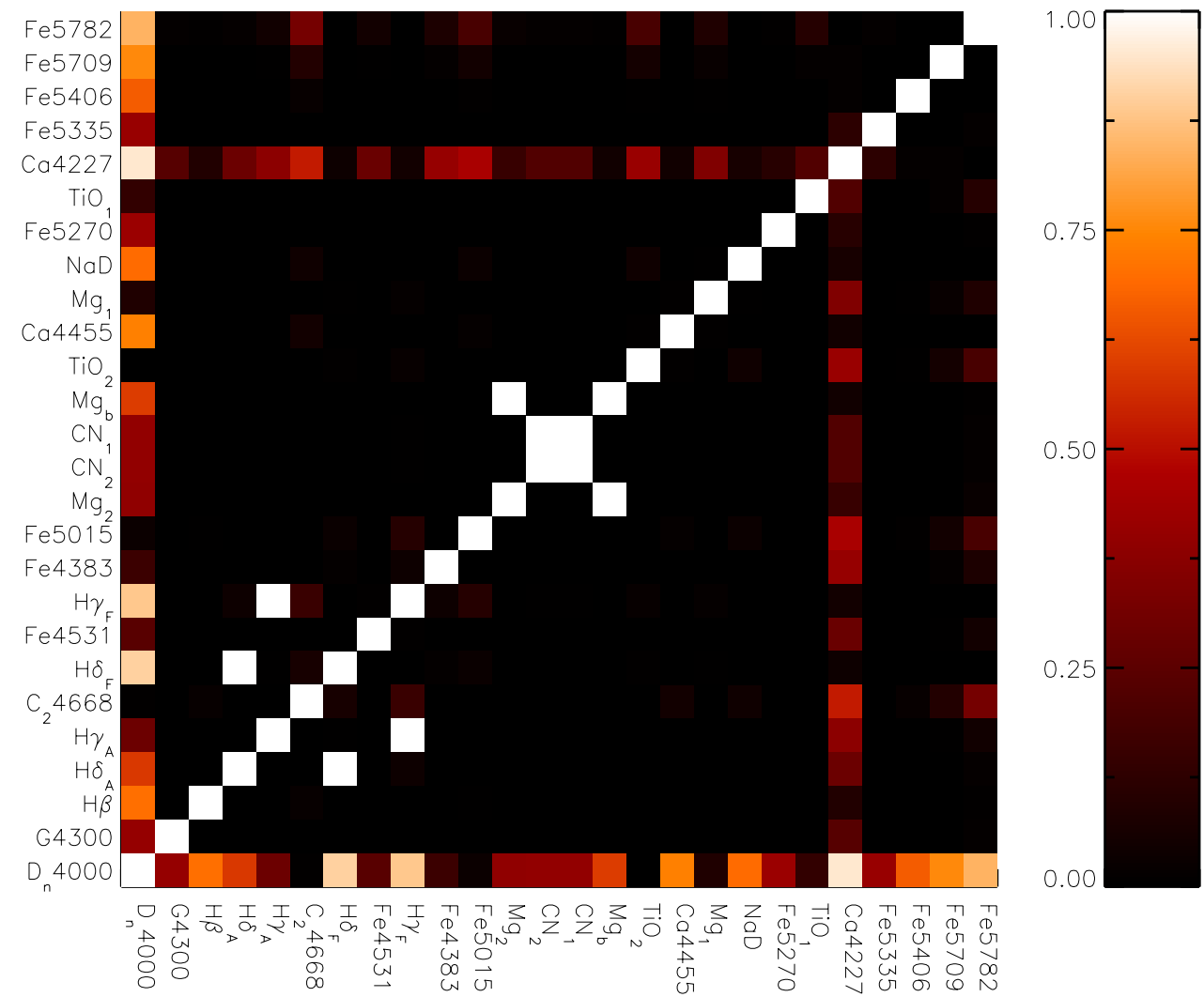

Figure 10. $\cos ^{2} \theta_{\mathrm{GNS}}$ between the subspaces spanned by the spectrum cutouts of the Extended Lick/IDS indicesin the SDSS configuration. The line indices are sorted by their LeverageScoreSum. Compared with the regions in Figure 9, we found fewer indices to be parallel to the $4000 \AA$ break. There is also a marked difference in the number of orthogonal index pairs.

(A color version of this figure is available in the online journal.)
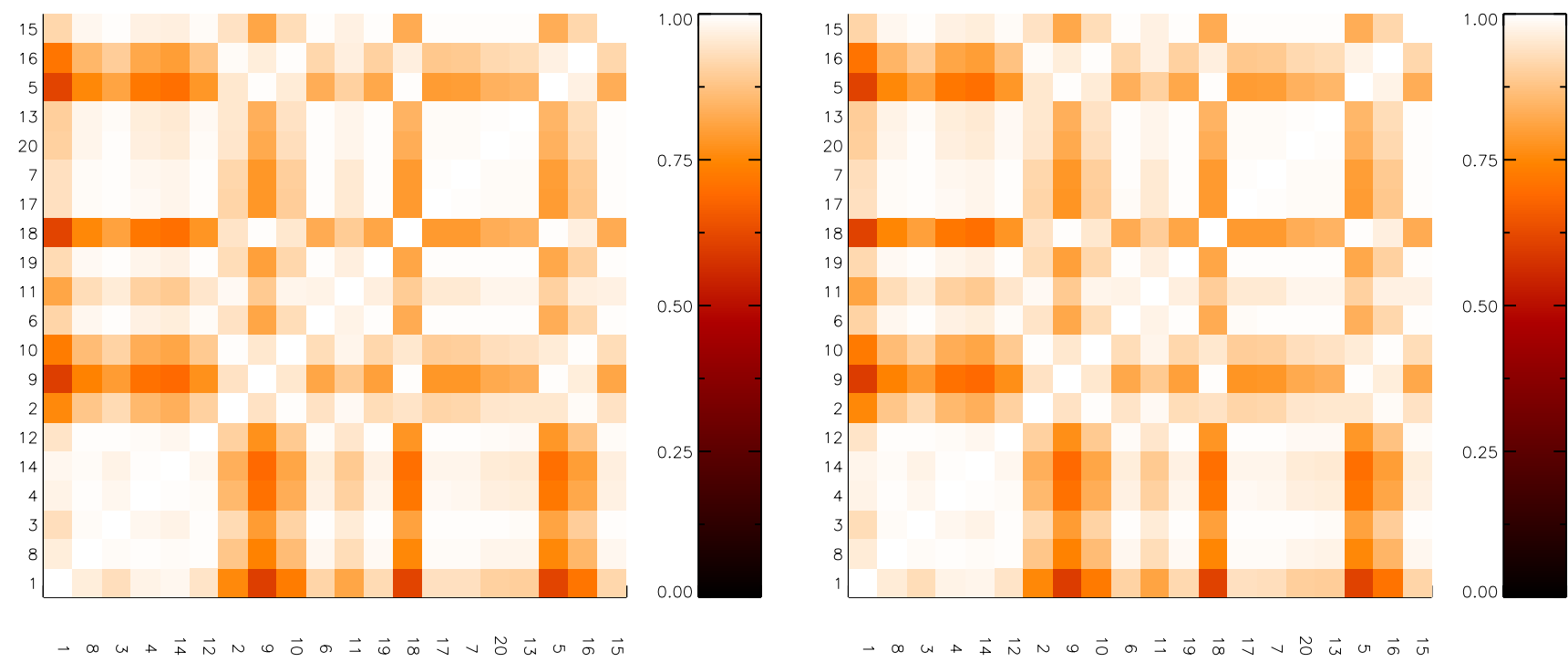

Figure 11. Left: squared Pearson correlation coefficient $\left(r^{2}\right)$ between the integrated flux of the identified regions. Right: the squared dot product between the first left-singular vectors of the spectral cutouts of the identified regions $\left(\cos ^{2} \theta_{\mathrm{PC} 1}\right)$. Both are given in the SDSS configuration. The diagonals show the auto-correlation of each region. We found both measures to be similar.

(A color version of this figure is available in the online journal.)

singular vectors. This result is not surprising, because the integrated flux does not fully describe a region, or in fact any spectrum. This result also justifies the use of the PCA to determine physical parameters from a galaxy spectrum. Interestingly, from Figure 11 we also find both measures to be similar. A mathematical explanation is however not yet explored.

\subsection{Physical Significance of Regions}

The parameter sensitivities of the regions are given in Columns 9 to 12 of Tables 2 and 3, respectively, for the Lick/IDS and SDSS configurations. All regions are sensitive to stellar age to the first order, and to either stellar age or stellar 

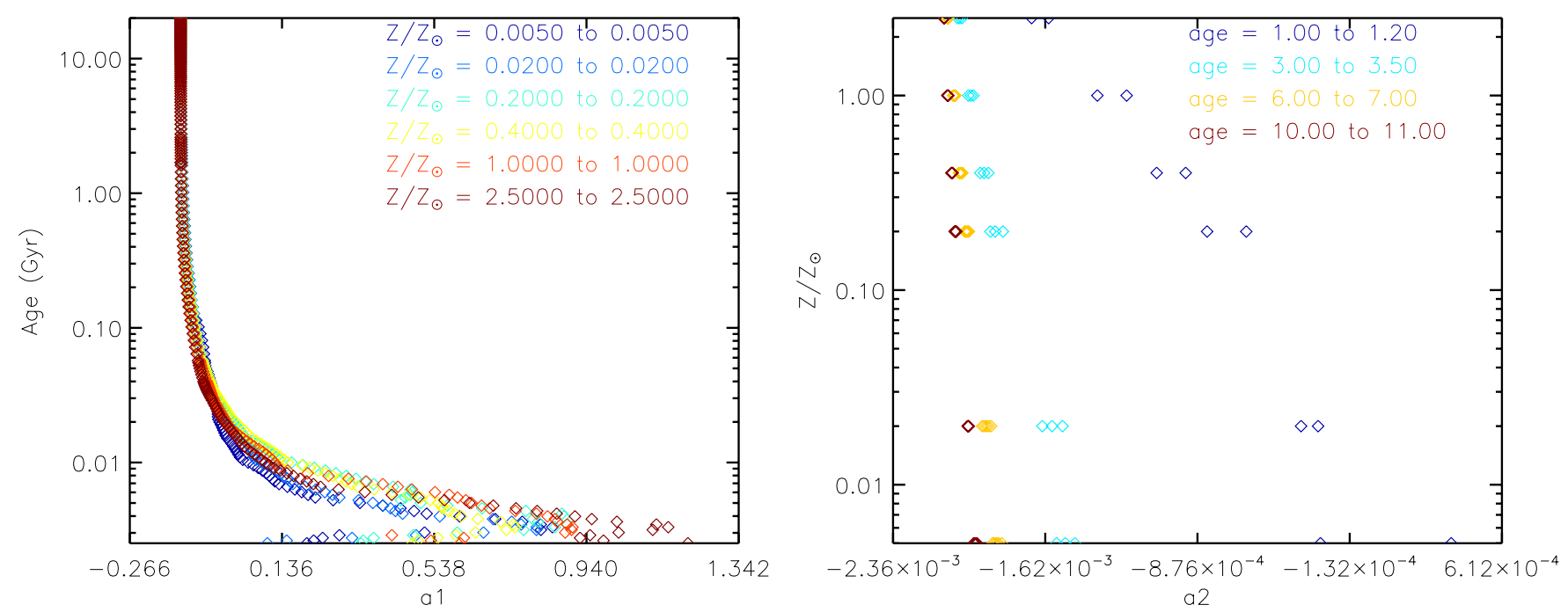

Figure 12. Left: dependence of stellar age on the first PCA eigencoefficient of Region01, for different stellar metallicities. Right: The dependence of stellar metallicity on the second PCA eigencoefficient, for different stellar ages, in the SDSS configuration. Although the relations are monotonic (except the smallest ages on the left), they are not straightly linear, which in turn explains the small correlation coefficient amplitude listed in Table 3 . This result suggests that other (nonlinear) measures, rather than the Pearson correlation coefficient, may better describe the relation between the eigencoefficients and the physical parameters.

(A color version of this figure is available in the online journal.)

metallicity to the second order. Sánchez Almeida et al. (2012) have also shown that age is the main parameter driving the variance in the spectra of local galaxies. We expect some regions will be sensitive to the stellar metallicity to the first order if the input spectra were continuum subtracted, taking out the sample variance that is primarily due to stellar age. While this is an interesting alternative to the data centering (in the preprocessing, Section 2), care must be taken to propagate the uncertainty of the estimated continuum into the region identification.

We then perform a PCA on the cutouts from Region01 in the SDSS configuration and relate the eigencoefficients to stellar age and metallicity. The eigenspectra are shown in Appendix B. Focusing on the lowest orders of eigencoefficients, shown in Figure 12, the first eigencoefficient correlates well with the stellar age regardless of the stellar metallicity, and the second eigencoefficient with the stellar metallicity after the age is known. These results agree with those obtained from the parameter sensitivity analysis that are shown in Columns 9 to 12 of Table 3 . The determination of stellar age from the first eigencoefficient appears to work best for intermediate ages, which is not surprising considering that the optical spectra of galaxies dominated by old ( $>$ a few Gyr) stellar populations look alike, posing a well-known limitation on the determination of stellar age in galaxies from optical spectra. We also found a similar situation for galaxies dominated by very young populations ( $<10 \mathrm{Myr})$, where spectra of two different ages can show the same firsteigencoefficient amplitude. Interestingly, we have to know the stellar age before we can tell the stellar metallicity; in other words, they have to be determined simultaneously, which is a manifestation of the well-known "age-metallicity degeneracy" problem (e.g., Faber 1973; Mould 1978; Schmidt et al. 1989) in galaxy parameter estimation. To conclude, we can use the regional eigencoefficients to determine the stellar age and metallicity in early-type galaxies that have solar abundance ratios, no dust, and a single-burst star formation history. It remains to be seen how the parameters that are relevant to late-type galaxies, such as the exponentially decreasing star formation rate and the dust extinction, depend on the eigencoefficients of a region.

\section{CONCLUSIONS}

We identify informative wavelength regions in a single-burst stellar population model using the CUR Matrix Decomposition (Drineas et al. 2008; Mahoney \& Drineas 2009). The regions are objective. They are shown to be sensitive to stellar age and metallicity. The regions can be used to determine the stellar age and metallicity in early-type galaxies that have solar abundance ratios, no dust, and a single-burst star formation history. The region identification method and the subspace analysis can be applied to any set of spectra of the user's interest, so that we eliminate the need for a common, fixed resolution index system.

We plan to extend this analysis to late-type galaxies. The presence of emission lines poses special challenges to the region identification on the whole spectra, namely, the continuum + absorption + emission spectra. This speculation is hinted at by the fact that the continuum-included strong emission lines cannot be reconstructed with higher than $\approx 20 \%$ accuracy on average by using a handful of lower-order eigenspectra (Yip et al. 2004a; Marchetti et al. 2012). We are investigating this question. A possible approach is that of Győry et al. (2011) who parameterized the continuum-subtracted emissionline EWs through a handful of eigenspectra. To this end, the SDSS galaxies will be a perfect data set. Because of the many galaxy types, a large diversity in the emission lines and the associated gas kinematics can be studied. A set of regions, taking into account both absorption and emission lines, will give a comprehensive parameterization to emission-line galaxy spectra. The progress toward understanding galaxy parameters has already set benchmarks in the field (e.g., Kauffmann et al. 2003; Gallazzi et al. 2005; Wild et al. 2007; Chen et al. 2012). This work provides a factor of $\approx 100$ reduction over the original data. Such a data compression will be crucial if we want to simultaneously estimate a large number $(>10)$ of galaxy parameters (Yip \& Wyse 2007; Yip 2010) in the future, such as stellar age and metallicity, dust extinction, and high temporal resolution star formation history. 


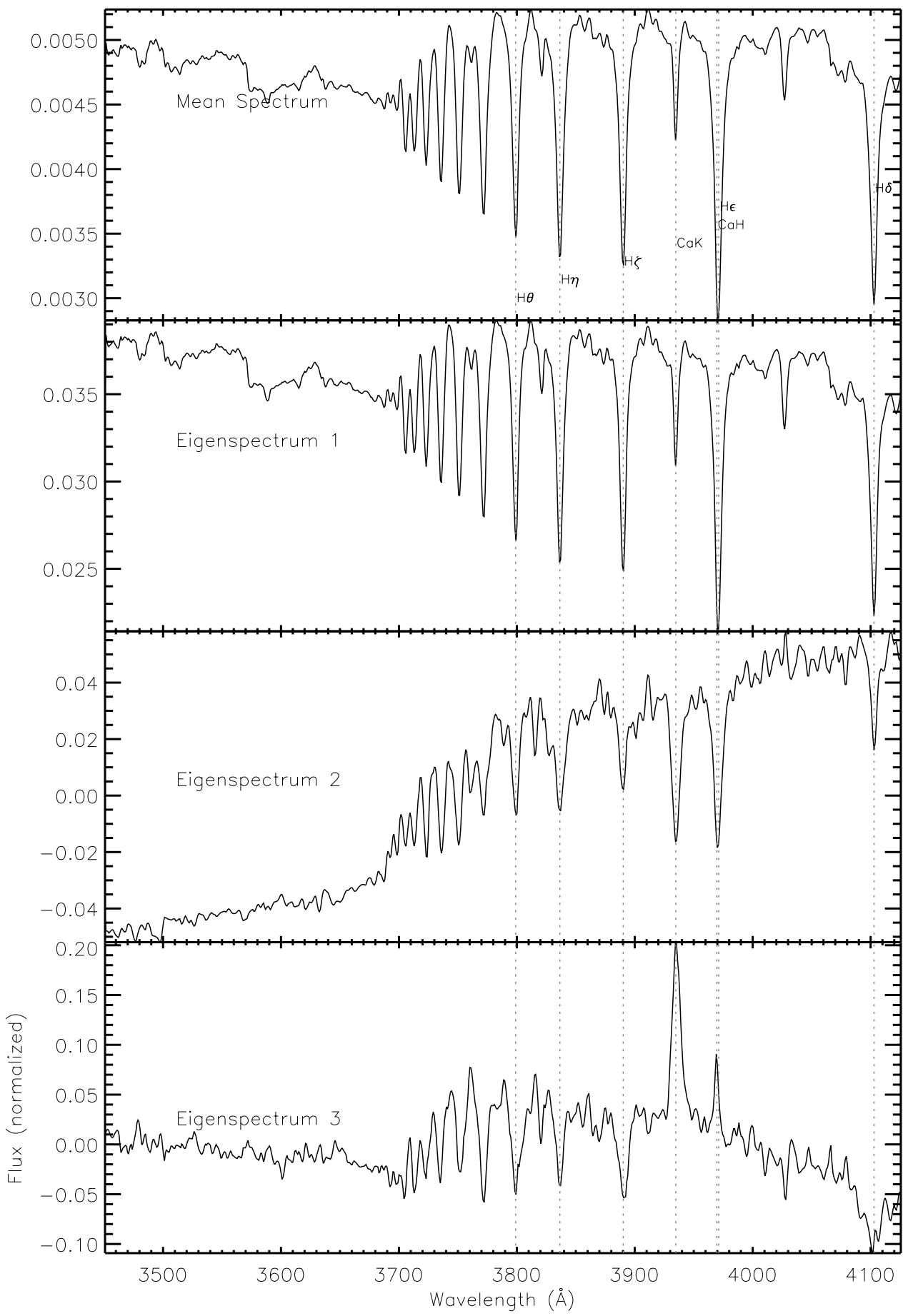

Figure 13. Mean spectrum (top) and first three eigenspectra of Region01 in the SDSS configuration. The first eigenspectrum modulates the Balmer absorption strength (i.e., the stellar age). The second eigenspectrum modulates the $4000 \AA$ break strength (i.e., the age-metallicity degeneracy). The third eigenspectrum shows an anti-correlation between the strengths of the Balmer and the $\mathrm{Ca} \mathrm{K}$ and $\mathrm{H}$ absorptions.

We thank Andrew Connolly, Haijun Tian, Miguel Angel Aragon Calvo, and Guangtun Zhu for useful comments and discussions. C.W.Y. thanks Scott Trager for discussions on galaxy spectra. We thank the referee for careful reading of the manuscript and useful suggestions.

This research is partly funded by the Gordon and Betty Moore Foundation through grant GBMF\#554.01 to the Johns Hopkins University. I.C. and L.D. acknowledge grant OTKA-103244. This research has made use of data obtained from or software provided by the U.S. National Virtual Observatory, which is sponsored by the National Science Foundation.

\section{APPENDIX A}

\section{CUR MATRIX DECOMPOSITION}

The CUR Matrix Decomposition (Drineas et al. 2008; Mahoney \& Drineas 2009) computes a low-rank approximation to an arbitrary matrix that marvels the optimal low-rank approximation provided by the truncated SVD. The approximation, however, is expressed in terms of a small number of actual columns and/or rows of the input data matrix. That is, it captures the dominant modes of variation in a data matrix with a small number of actual (and thus potentially 
interpretable) columns and/or rows, rather than a small number of (in general non-interpretable) eigencolumns and eigenrows. The CUR Matrix Decomposition has been used in genetics to identify "PCA-correlated single-nucleotide polymorphisms (SNPs)," basically the most informative columns within Deoxyribonucleic acid SNP matrices (Paschou et al. 2007), and it has been central to recent work in developing randomized algorithms for the low-rank approximation of very large matrices (Mahoney 2012).

The main idea behind the CUR Matrix Decomposition is to decompose a given matrix $A$ into matrices $C$ and $R$, which consist of, respectively, a small number of actual columns and rows of $A$, and a low-dimensional encoding matrix $\mathbb{U}$, such that $A \approx C U R$ as follows

$$
\|A-C U R\|_{F} \leqslant(1+\epsilon)\left\|A-A_{k}\right\|_{F},
$$

where $A_{k}$ is the best rank $k$ approximation to $A$, as given by the truncated SVD. The fractional error of the decomposition is $\epsilon$. Subscript $F$ denotes the Frobenius norm of a matrix. We use only the column part of the decomposition in this paper. The choice of column is critical: to obtain low-rank approximation bounds of the form in Equation (A1), one chooses columns randomly according to an importance sampling distribution that is proportional to the Leverage Scores (of $A$, relative to the best rank $k$ approximation to $A$ ). These quantities, given in Equation (2), equal the diagonal elements of the projection matrix onto the span of the best rank $k$ approximation to $A$. They have been used previously in regression diagnostics as a measure of the importance or influence a given data point has on the least-squares fit (Chatterjee \& Hadi 1986). When applied to low-rank matrix approximations, the Leverage Scores provide a measure of how informative is a given column to the best rank $k$ approximation of the data matrix. In our case, a column is a wavelength in the model spectra.

\section{APPENDIX B}

\section{REGIONAL EIGENSPECTRA}

The lowest orders of the eigenspectra encapsulate most of the sample variance in the full optical spectra of nearby galaxies (Yip et al. 2004a), forming a subspace that lies in a higherdimensional wavelength space. We examine here the first few orders of eigenspectra of Region01 in the SDSS configuration. Together with the mean spectrum of the model the eigenspectra are plotted in Figure 13. The third eigenspectrum shows an anti-correlation between the strengths of the Balmer and the $\mathrm{Ca} \mathrm{K}$ and $\mathrm{H}$ absorptions. Wild et al. (2007) also found that the third mode modulates the $\mathrm{Ca} \mathrm{K}$ and $\mathrm{H}$ strength, though the concerned model has an exponential star formation history with recent stellar bursts. We see from Figure 13 that the first and second eigenspectra modulate, respectively, the Balmer absorption and the $4000 \AA$ break strengths. On the other hand, we know from the parameter dependence in Figure 12 that first eigenspectrum of the single-burst stellar population model tells primarily the stellar age. The second eigenspectrum is related to the age-metallicity degeneracy. We therefore conclude the following. The larger the first eigencoefficient of a galaxy spectrum, the stronger the Balmer absorptions, indicating that the stellar populations are younger. The larger the second eigencoefficient, the stronger the $4000 \AA$ break, indicating that the stellar populations are either older and less metal-rich, or younger and more metal-rich.

\section{REFERENCES}

Balogh, M. L., Morris, S. L., Yee, H. K. C., Carlberg, R. G., \& Ellingson, E. 1999, ApJ, 527, 54

Bruzual, A. G. 1983, ApJ, 273, 105

Bruzual, G., \& Charlot, S. 2003, MNRAS, 344, 1000

Budavári, T., Wild, V., Szalay, A. S., Dobos, L., \& Yip, C.-W. 2009, MNRAS, 394, 1496

Burstein, D., Faber, S. M., Gaskell, C. M., \& Krumm, N. 1984, ApJ, 287, 586

Chabrier, G. 2003, PASP, 115, 763

Chatterjee, S., \& Hadi, A. S. 1986, StaSc, 1, 379

Chen, Y.-M., Kauffmann, G., Tremonti, C. A., et al. 2012, MNRAS, 421,314

Connolly, A. J., Szalay, A. S., Bershady, M. A., Kinney, A. L., \& Calzetti, D. 1995, AJ, 110, 1071

Dobos, L., Csabai, I., Yip, C.-W., et al. 2012, MNRAS, 420, 1217

Drineas, P., Mahoney, M. W., \& Muthukrishnan, S. 2008, SIAM. J. Matrix Anal., Appl., 30, 844

Faber, S. M. 1973, ApJ, 179, 731

Faber, S. M., Friel, E. D., Burstein, D., \& Gaskell, C. M. 1985, ApJS, 57, 711

Francis, P. J., Hewett, P. C., Foltz, C. B., \& Chaffee, F. H. 1992, ApJ, 398, 476

Gallazzi, A., Charlot, S., Brinchmann, J., White, S. D. M., \& Tremonti, C. A. 2005, MNRAS, 362, 41

Girardi, L., Bressan, A., Chiosi, C., Bertelli, G., \& Nasi, E. 1996, A\&AS, 117,113

Gunawan, H., Newwan, O., \& Setya-Budhi, W. 2005, Contrib. Algebra Geom., 46, 331

Győry, Z., Szalay, A. S., Budavári, T., Csabai, I., \& Charlot, S. 2011, AJ, 141,133

Jones, L. A., \& Worthey, G. 1995, ApJL, 446, L31

Kauffmann, G., Heckman, T. M., White, S. D. M., et al. 2003, MNRAS, 341,33

Krughoff, K. S., Connolly, A. J., Frieman, J., et al. 2011, ApJ, 731, 42

Marchetti, A., Granett, B. R., Guzzo, L., et al. 2012, MNRAS, 428, 1424

Madgwick, D. S., Hewett, P. C., Mortlock, D. J., \& Wang, L. 2003, ApJL, 599, L33

Mahoney, M. W. 2012, in Advances in Machine Learning and Data Mining for Astronomy, ed. M. J. Way et al. (Hoboken, NJ: CRC Press), 647

Mahoney, M. W., \& Drineas, P. 2009, PNAS, 106, 697

Mould, J. R. 1978, ApJ, 220, 434

Paschou, P., Ziv, E., Burchard, E. G., et al. 2007, PLoS Genetics, 3, 1672

Sánchez Almeida, J., Terlevich, R., Terlevich, E., Cid Fernandes, R., \& MoralesLuis, A. B. 2012, ApJ, 756, 163

Schmidt, A. A., Bica, E., \& Dottori, H. A. 1989, MNRAS, 238, 925

Shang, Z., Wills, B. J., Robinson, E. L., et al. 2003, ApJ, 586, 52

Strang, G. 1988, Linear Algebra and Its Applications (3rd ed.; San Diego, CA: Harcourt Brace Jovanovich Publishers)

Thomas, D., Maraston, C., \& Korn, A. 2004, MNRAS, 351, L19

Trager, S. C., Worthey, G., Faber, S. M., Burstein, D., \& Gonzalez, J. J. 1998, ApJS, 116, 1

Vanden Berk, D. E., Shen, J., Yip, C.-W., et al. 2006, AJ, 131, 84

Wild, V., \& Hewett, P. C. 2005, MNRAS, 358, 1083

Wild, V., Kauffmann, G., Heckman, T., et al. 2007, MNRAS, 381, 543

Worthey, G., Faber, S. M., \& Gonzalez, J. J. 1992, ApJ, 398, 69

Worthey, G., Faber, S. M., Gonzalez, J. J., \& Burstein, D. 1994, ApJS, 94, 687

Worthey, G., \& Ottaviani, D. L. 1997, ApJS, 111, 377

Yip, C.-W. 2010, AJ, 139, 342

Yip, C. W., Connolly, A. J., Szalay, A. S., et al. 2004a, AJ, 128, 585

Yip, C. W., Connolly, A. J., Vanden Berk, D. E., et al. 2004b, AJ, 128, 2603

Yip, C.-W., \& Wyse, R. F. G. 2007, in IAU Sym. 241, Stellar Populations as Building Blocks of Galaxies, ed. A. Vazdekis \& R. F. Peletier (Cambridge: Cambridge University Press), 533

York, D. G., Adelman, J., Anderson, J. E., Jr., et al. 2000, AJ, 120, 1579 